\title{
Desempenho, características da carcaça e da carne de bovinos $1 / 2$ sangue da raça Flamenga, terminados em pastagem de azevém anual e suplementados com casca de soja
}

\author{
Vanessa Ruiz Fávaro' ${ }^{1}$ Maicon Gaissler Lorena Pinto ${ }^{2}$, Diego de Córdova Cucco ${ }^{3}$, Simone Silmara Werner ${ }^{4}$ e Lucas Rossetto ${ }^{5}$
}

Resumo - O objetivo foi avaliar o desempenho e as características da carcaça e carne de novilhos de corte, sob pastejo de azevém anual com e sem suplementação energética com casca de soja. Foram utilizados 16 bovinos $1 / 2$ sangue da raça Flamenga castrados e distribuídos em dois tratamentos: 1) controle, alimentação exclusiva em pastagem de azevém anual; 2) alimentação em pastagem de azevém anual e suplementação com casca de soja a $0,6 \%$ do peso vivo. A idade e o peso médios inicial dos animais foram de 21 meses e 417,5kg, respectivamente. Os animais foram abatidos ao atingirem, no mínimo, $3 \mathrm{~mm}$ de espessura de gordura subcutânea (EGS), aos 26 meses de idade. Não foram verificados efeitos significativos dos tratamentos para as variáveis de desempenho e características da carcaça e da carne $(P>0,05)$. Animais $1 / 2$ sangue da raça Flamenga apresentaram carcaças com peso superior a $300 \mathrm{~kg}$. A terminação de bovinos em pastagem de azevém anual cv. Winter Star proporcionou carcaças com padrão de qualidade exigido pelos frigoríficos e consumidores com valores adequados de EGS, pH, maciez e coloração da carne e da gordura.

Termos para indexação: qualidade da carne; suplementação a pasto; terminação de bovinos

\section{Performance, carcass and meat characteristics of $1 \frac{1}{2}$ Flemish breed cattle, finished in annual ryegrass pasture, supplemented with soybean hulls}

\begin{abstract}
The objective was to evaluate the performance, meat and carcass characteristics of beef steers, grazing annual ryegrass with and without soybean hulls supplementation. Sixteen $1 / 2$ Flemish bovines, castrated, were distributed in two treatments: 1) control, exclusively fed with annual ryegrass pasture; 2 ) annual ryegrass pasture supplementation with soybean hulls at $0.6 \%$ of live weight. The animals' average age and initial weight were 21 months and $417.5 \mathrm{~kg}$, respectively. The animals were slaughtered when they reached at least $3 \mathrm{~mm}$ cover fat thickness (CFT), at 26 months of age. There were no significant effects of treatments neither on performance nor on carcass and meat characteristics $(P>0.05) .1 / 2$ Flemish blood steers presented carcasses weighing more than $300 \mathrm{~kg}$. Finishing in annual ryegrass pasture cv. Winter Star provided carcasses with the quality standards required by slaughterhouses and consumers with adequate values for $\mathrm{CFT}$, $\mathrm{pH}$, tenderness and color of meat and fat.
\end{abstract}

Index terms: finishing cattle; meat quality; pasture supplementation

\section{Introdução}

O sistema de terminação de bovinos de corte em pastagem geralmente, caracteriza-se pelo baixo desempenho dos animais e pouca energia ingerida, resultando em elevada idade de abate e carcaças com acabamento ausente ou escasso (RESENDE et al., 2014). Na Região Sul do Brasil, as forrageiras anuais de clima temperado são alternativas para suprir o déficit alimentar durante o inverno e a primavera. $O$ azevém anual é uma das forrageiras mais utilizadas devido à sua rusticidade, longo período de oferta e alto valor nutritivo (RODRIGUES et al., 2011). Gramíneas anuais de inverno normalmente têm alta digestibilidade e altos teores de nitrogênio (N) degradável (SILVEIRA et al., 2006), o que pode gerar um desequilíbrio entre os teores de proteína e energia em relação às concentrações ideais para atender às exigências nutricionais de bovinos. O excesso de proteína representa custo energético para o animal (para eliminação da amônia através do ciclo da ureia) e tem um impacto negativo sobre o meio ambiente, uma vez que aumenta a excreção de nitrogênio na urina e nas fezes (BATISTA et al., 2017). A suplementação energética, em pastagens com alto teor proteico, é uma alternati-

Recebido em 5/11/2020. Aceito para publicação em 15/1/2021.

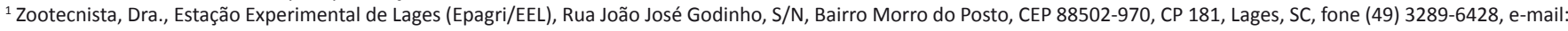
vanessafavaro@epagri.sc.gov.br

${ }^{2}$ Médico-veterinário, Dr., Epagri/EEL, e-mail: maiconpinto@epagri.sc.gov.br

${ }^{3}$ Médico-veterinário, Dr., Universidade do Estado de Santa Catarina (Udesc/CEO), Rua Beloni Trombeta Zanin, 680-E. Bairro Santo Antônio. CEP: 89815-630. Chapecó, SC fone: (49) 2049-9561, e-mail: diego.cucco@udesc.br.

${ }^{4}$ Matemática, Dra., Epagri/EEL, e-mail: simonewerner@epagri.sc.gov.br

${ }^{5}$ Acadêmico do curso de Zootecnia (Udesc/CEO), e-mail: Irossetto6@gmail.com 
va para aumentar a velocidade de crescimento dos animais através do melhor balanceamento dos nutrientes da dieta e elevar a produção animal por unidade de área (HELLBRUGGE et al., 2008).

A maioria dos estudos sobre suplementação energética em pastagens hibernais avaliou o fornecimento de miIho triturado que, historicamente, tem o preço mais elevado em comparação a outras opções como a casca de soja. A casca de soja, por apresentar alto conteúdo de fibra efetiva, estimula a salivação e ruminação e, mantém o ambiente ruminal satisfatório (TAMBARA et al., 1995). Santos et al. (2005) avaliaram o desempenho de novilhas de corte em pastagem de aveia-preta e azevém recebendo suplementação com milho ou casca de soja e verificaram que a suplementação com casca de soja proporcionou ganho médio diário semelhante ao tratamento com milho e superior à alimentação exclusiva em pastagem. Objetivou-se com esse estudo avaliar o desempenho e características da carcaça e da carne de novilhos de corte, $1 / 2$ sangue da raça Flamenga, sob pastejo em azevém anual com e sem suplementação energética com casca de soja.

\section{Material e métodos}

O ensaio foi realizado em uma área experimental de oito hectares semeada com azevém anual tetraploide cv. Winter Star, com densidade de sementes de $25 \mathrm{~kg} \mathrm{ha}^{-1}$. A adubação, no momento da semeadura, consistiu de $200 \mathrm{~kg} \mathrm{ha}^{-1}$ da fórmula comercial NPK 05-25-25. A adubação de cobertura, à base de ureia, foi de $100 \mathrm{~kg}$ de $\mathrm{N} \mathrm{ha}^{-1}$, dividida igualmente em duas aplicações. O período de pastejo foi de 16 de agosto a 9 de dezembro de 2017, dividido em quatro subperíodos: I (16/08/2017 a 13/09/2017), II (14/09/2017 a 12/10/2017), III $(13 / 10 / 2017$ a $10 / 11 / 2017)$ e IV (11/11/2017 a 09/12/2017).

Foram utilizados 16 bovinos machos castrados, provenientes do cruzamento entre vacas mestiças e touro da raça Flamenga, com idade média de 21 meses e peso médio inicial de $417,5 \mathrm{~kg}$. 0 método de pastejo foi de lotação contínua e carga fixa, com taxa de lotação média de 2,29UA ha-1. Os animais foram distribuídos ao acaso em dois tratamentos: pastagem de azevém anual sem suplementação e pastagem de azevém anual com suplementação energética. Durante todo o período experimental foi fornecida mistura mineral comercial e água ad libitum. A disponibilidade média de forragem foi de $1.728,3 \mathrm{~kg}$ de MS ha-1. A forragem apresentou, em média $17,6 \%$ de proteína bruta $(\mathrm{PB})$, $64,5 \%$ de digestibilidade in vitro da matéria orgânica (DIVMO), 58,6\% de fibra em detergente neutro (FDN) e 31,2\% de fibra em detergente ácido (FDA). A suplementação energética foi à base de casca de soja ( $0,6 \%$ do peso vivo), fornecida diariamente às 10 h00 (composição bromatológica: $10,6 \%$ de PB; $68,8 \%$ de DIVMO; 70,3\% de FDN e 52,1\% de FDA).

Para determinação do desempenho e carga animal (CA), os animais foram pesados, em jejum hídrico e alimentar prévio de 12 horas, a cada 28 dias. 0 ganho de peso por hectare (GPA) foi obtido multiplicando-se o número de animais $\mathrm{dia}^{-1} \mathrm{ha}^{-1}$ pelo ganho de peso diário (GPD) dos animais, multiplicado pelo número de dias de cada período. No mesmo momento das pesagens foram realizadas mensurações por ultrassonografia da espessura de gordura subcutânea (EGS), entre a 12a e 13a costelas, transversal ao músculo Longissimus thoracis, e a EGS medida no terço médio distal do mesmo músculo. Os animais foram abatidos quando a EGS média do grupo foi igual ou superior a $3 \mathrm{~mm}$. As carcaças foram divididas longitudinalmente ao meio e pesadas para obtenção do peso da carcaça quente (PCQ), e o rendimento de carcaça quente (RCQ) foi obtido pela relação entre o peso de carcaça quente e o peso de abate. Após o abate, as meias-carcaças foram armazenadas a $4^{\circ} \mathrm{C}$ durante 24 horas, quando foram novamente pesadas para a obtenção do peso de carcaça fria (PCF) e calculada a perda por resfriamento (PR). No momento do abate e 24 horas após foi aferido o pH com peagâmetro digital com eletrodo de penetração e ainda mensurada a EGS com paquímetro digital, todas medidas na região da 12 a costela da meia carcaça esquerda.

Após as medições, foi retirada, da meia carcaça esquerda, uma seção do músculo Longissimus, compreendida entre a $12^{a}$ e $13^{a}$ costelas, dividido em três bifes com 2,54 centímetros de espessura. $A$ área de olho de lombo ( $A O L$ ) foi mensurada com auxílio do software Image ${ }^{\circledR}$. A determinação da cor do músculo e da gordura foi realizada 24 horas após o abate (TAPP III et al., 2011), utilizando o colorímetro Minolta (modelo CR 400, Minolta Camera Co. Ltd. Osaka, Japão). Obtiveram-se os valores de luminosidade ( $\mathrm{L}^{*}=0$ preto, 100 branco), a* em que o índice varia de verde (-) para vermelho $(+)$ e $b^{*}$ em que o índice varia de azul (-) para amarelo (+). Os cálculos de perdas por cocção (PC) foram determinados pelos pesos dos bifes antes e depois da cocção, após a temperatura interna da amostra até atingir $75^{\circ} \mathrm{C}$ no seu centro geométrico. Para determinação da força de cisalhamento (FC) foram retirados seis cilindros, com $1,27 \mathrm{~cm}$ de diâmetro, paralelos ao eixo longitudinal das fibras musculares (AMSA, 2015). Os cilindros foram completamente cortados perpendicularmente às fibras musculares com lâmina de Warner-Bratzler através de texturômetro (Texture Analyser TA-XT2I). Foi então calculada a média de força de corte dos cilindros para representar a FC de cada amostra.

O delineamento experimental foi casualizado em blocos. Foram utilizados dois blocos, 4 parcelas por bloco (2 parcelas para o tratamento controle e 2 parcelas para o tratamento com suplementação) e dois animais testers por parcela, totalizando dezesseis animais. Os dados coletados foram submetidos à análise de variância, sendo verificadas as pressuposições de homogeneidade de variância (teste de Bartlett) e normalidade dos resíduos (teste de shapiro-wilk). Para os dados de desempenho foi realizada análise de regressão polinomial, em função dos períodos de avaliação e, verificada interação entre tratamento e período. Para os dados de carne e carcaça o peso inicial dos animais foi considerado como covariável. Todas as análises foram realizadas considerando o nível de $5 \%$ de significância por meio do programa estatístico $R$ ( $R$ Core Team, 2018).

\section{Resultados e discussão}

Os valores de GPD, CA e GPA foram semelhantes entre os tratamentos (em 
média: $1,35 \mathrm{~kg} \mathrm{dia}^{-1} ; 1031,7 \mathrm{~kg} \mathrm{ha}^{-1} \mathrm{e}$ $80,4 \mathrm{~kg} \mathrm{ha}^{-1}$, respectivamente). Também não foi verificada interação significativa entre os tratamentos e os períodos de avaliação $(P>0,05)$. Dessa forma, na Tabela 1 estão apresentadas as médias em função dos períodos de avaliação.

Era esperado ganho de peso individual superior para os animais alimentados com casca de soja, pois o incremento no consumo de energia resultaria em melhor proporção entre NDT e PB. Corroborando com os resultados, Lazzarotto et al. (2019) verificaram ganhos de pesos semelhantes em novilhos mantidos em pastagem de aveia e azevém suplementados com milho triturado, fato atribuído ao efeito substitutivo quando a suplementação é usada em pastagens temperadas. Moore et al. (1999) afirmaram que o efeito da suplementação sobre o ganho de peso diário é mais evidenciado em pastagens de baixa qualidade, como as pastagens tropicais. 0 GPD e GPA oscilaram de acordo com os períodos de pastejo $(P<0,05)$, e foram mais bem representados pela equação quadrática (Tabela 1), com desempenho crescente até o 68잉. A CA foi crescente ao longo do tempo, representada por equação linear em função do aumento do peso vivo dos animais. Os pastos cultivados de inverno têm como característica alta digestibilidade. No entanto, a concentração de matéria seca (MS) na fase inicial de crescimento é baixa e, já na metade final do ciclo de produção, o nível energético declina em decorrência de mudanças na estrutura da pastagem com maior proporção de colmos no final do ciclo das forrageiras (PILAU et al., 2003). Dessa forma, o ganho médio diário dos animais em pastejo não é uniforme, mesmo mantendo oferta de forragem adequada.

O peso final dos animais, os pesos das carcaças quente e fria e o rendimento de carcaça quente não foram influenciados pelos tratamentos $(P>0,05$; Tabela 2). Pode-se observar que o peso de abate foi superior a $570 \mathrm{~kg}$ resultando em carcaças com mais de $300 \mathrm{~kg}$, valor acima do mínimo estabelecido pelos frigoríficos de $225 \mathrm{~kg}$ (MOLETTA et al., 2014). O tipo racial utilizado se enquadra em um grupo genético de grande porte que, quando bem alimentado, atinge peso e acabamento desejado,
Tabela 1. Ganho de peso diário (GPD), carga animal (CA) e ganho de peso por hectare (GPA) de bovinos em pastagem de azevém anual, em função dos períodos de avaliação

Table 1. Daily weight gain (DWG), animal stocking (AS) and weight gain per area (WGA) of steers in annual ryegrass pasture, depending on the evaluation periods

\begin{tabular}{|c|c|c|c|c|c|}
\hline \multirow{2}{*}{ Tratamentos } & \multicolumn{4}{|c|}{ Períodos de avaliação } & \multirow{2}{*}{ Equações de regressão } \\
\hline & 1 & II & III & IV & \\
\hline GPD, kg dia-1 & 1,22 & 1,52 & 1,51 & 1,17 & $Y=-0,16 x^{2}+0,78 x+0,6$ \\
\hline $\mathrm{CA}, \mathrm{kg} \mathrm{ha}^{-1}$ & 903,6 & 989,1 & 1077,2 & 1156,9 & $Y=84,82 x+819,67$ \\
\hline GPA, kg ha-1 & 68,4 & 85,5 & 88,1 & 79,7 & $Y=-6,36 x^{2}+35,44 x+39,53$ \\
\hline
\end{tabular}

com ótimo rendimento de carcaça. $O$ RCQ médio foi de $53,5 \%$, segundo Restle et al. (2001), quando são utilizados volumosos com alta taxa de passagem, como o azevém, não são encontradas diferenças no rendimento de carcaça por não haver diferença no conteúdo gastrointestinal dos animais. A espessura de gordura subcutânea e a perda por resfriamento foram semelhantes entre os tratamentos $(P>0,05)$. A EGS é um dos principais parâmetros para avaliar a carcaça bovina e ponto de referência para classificação e pagamento da carcaça nos principais frigoríficos brasileiros (ROCHA, 1999). A EGS obtida nesse estudo ficou acima de $3 \mathrm{~mm}$, valor mínimo exigido pelos frigoríficos. A semelhança entre as espessuras de gordura subcutânea pode justificar a semelhança $(P>0,05)$ para a perda por resfriamento entre os tratamentos, já que a gordura tem como principal função a proteção da carcaça contra a desidratação e o escurecimento da parte externa dos músculos durante o resfriamento (MULLER, 1987). A AOL média foi de $105,9 \mathrm{~cm}^{2}$ (P>0,05). Valores mais elevados de AOL são observados quando se utiliza cruzamento com raças de grande porte. Ribeiro et al. (2002) avaliaram animais cruzados $3 / 4$ Bos taurus taurus e $1 / 4$ Bos taurus indicus e obtiveram $106,3 \mathrm{~cm}^{2}$ de AOL.
Tabela 2. Peso inicial e final, peso da carcaça quente (PCQ), peso da carcaça fria (PCF), rendimento da carcaça quente (RCQ), perda por resfriamento (PR), espessura de gordura de cobertura (EGC), área de olho de lombo (AOL), força de cisalhamento (FC) e perda por cocção (PC), da carcaça de bovinos mantidos em pastagem de azevém anual, com ou sem suplementação energética ( $0,6 \%$ do peso vivo)

Table 2. Initial and final weight, hot carcass weight (HCW), cold carcass weight (CCW), hot carcass yield $(\mathrm{HCY})$, cooling loss $(\mathrm{CL})$, cover fat thickness (CFT), rib eye area (REA), shear force (SF) and cooking loss (CL), of the carcass of cattle grazing annual ryegrass pasture, with or without energy supplementation ( $0.6 \%$ of live weight)

\begin{tabular}{lccc}
\hline Item & Controle & Suplemento & Valor de $p$ \\
\cline { 1 - 3 } Peso inicial, kg & $416,2 \pm 11,94$ & $418,8 \pm 13,03$ & - \\
Peso final, kg & $574,6 \pm 20,00$ & $594,7 \pm 14,68$ & 0,428 \\
PCQ, kg & $305,7 \pm 12,67$ & $314,1 \pm 12,12$ & 0,645 \\
PCF, kg & $300,7 \pm 12,43$ & $310,0 \pm 12,51$ & 0,614 \\
RCQ, \% & $53,2 \pm 0,52$ & $53,9 \pm 0,84$ & 0,457 \\
PR, \% & $1,66 \pm 0,25$ & $1,38 \pm 0,28$ & 0,486 \\
EGC, mm & $4,33 \pm 0,56$ & $4,52 \pm 0,59$ & 0,819 \\
AOL, cm ${ }^{2}$ & $103,8 \pm 6,38$ & $108,0 \pm 5,37$ & 0,625 \\
FC, kgf cm ${ }^{-2}$ & $3,09 \pm 0,26$ & $3,58 \pm 0,39$ & 0,331 \\
\hline PC, \% & $17,14 \pm 1,39$ & $16,78 \pm 1,38$ & 0,859 \\
\hline pH, (Oh) & $6,55 \pm 0,10$ & $6,34 \pm 0,06$ & 0,074 \\
pH, (24h) & $5,14 \pm 0,06$ & $5,11 \pm 0,05$ & 0,688 \\
\hline
\end{tabular}


A maciez é uma das qualidades mais desejáveis na carne, do ponto de vista do consumidor. Segundo Oliveira et al. (2012) a carne bovina é classificada como macia por valores de força de cisalhamento inferior a $4,5 \mathrm{kgf} \mathrm{cm}^{-2}$ e neste estudo o valor médio obtido foi de $3,33{\mathrm{kgf} \mathrm{cm}^{-2}}^{-}(P>0,05)$. Diversos fatores influenciam positivamente na maciez da carne, entre eles o cruzamento com Bos taurus. Comparando Bos indicus e o cruzamento Bos taurus $\times$ Bos indicus, Andrade et al. (2010) observaram maior maciez da carne dos animais cruzados. De acordo com Pitombo et al. (2013) menores perdas por cocção contribuem para carne mais macia e suculenta. A perda de peso média devido à cocção não diferiu entre os tratamentos (média de $16,7 \%$ ). Corroborando com os resultados, Maggioni et al. (2012) obtiveram valores semelhantes de maciez $(2,96 \mathrm{kgf}$ $\mathrm{cm}^{-2}$ ) e perda por cocção $(16,99 \%)$ ao avaliarem o cruzamento de bovinos da raça Nelore com Limousin. A avaliação do $\mathrm{pH}$ final da carcaça é importante, pois é responsável por alterações nas características de qualidade da carne, como a cor, a maciez, a textura e a capacidade de retenção de água (RESENDE et al., 2014). As médias de $\mathrm{pH}$ final obtidas $(5,12)$ foram inferiores a 5,8 que, conforme Gregory (1998), seria o valor limite para a ocorrência de carnes tipo DFD (dark, firm and dry), sendo assim o produto final considerado adequado para manutenção da vida de prateleira. A cor da carne é uma característica determinante para compra pelo consumidor, visto que colorações mais escuras são associadas à possível deterioração. O valor de $\mathrm{pH}$ após o abate está diretamente relacionado com a luminosidade e a coloração da carne. Nesse estudo os valores de $\mathrm{pH}$ permaneceram dentro dos limites ideais e, consequentemente, as características de $L^{*}$, a* e b* enquadraram-se dentro da normalidade (Tabela 3). Muchenje et al. (2009) descreveram que, em bovinos, os índices de luminosidade variam entre 33,2 - 41,0, a cor vermelha entre 11,1 - 23,6 e a cor amarela, entre 6,1 - 11,3. A coloração da carne está associada à idade de abate, havendo correlação negativa entre a cor e a idade de abate dos animais (MISSIO et al., 2010), reflexo da maior quantidade, em animais jovens, do pigmento mioglobina (responsável pela cor avermelhada da carne). Nesse estudo os animais foram abatidos com idade média de 26 meses. A cor da gordura é influenciada pelo conteúdo de carotenoides derivados das plantas. Pastagens verdes e frescas geralmente contêm quantidades elevadas de carotenoides (até 500ppm de matéria seca) (REALINI et al., 2004). O pigmento amarelo da gordura normalmente está associado ao animal produzido no pasto, enquanto a gordura menos pigmentada (branca) está relacionada a animais terminados em confinamento. Fernandes et al., (2008) observaram que animais confinados, alimentados com silagem de milho e concentrado, apresentaram menor intensidade do croma $b^{*}(7,20)$ em relação ao presente estudo $(10,15$ em média).

Os sistemas de produção devem utilizar materiais genéticos apropriados aos seus objetivos. A utilização de animais de raças ou cruzamentos de grande porte será viável se as condições ambientais, principalmente a nutrição, permitirem, porque são animais que têm requerimentos nutricionais elevados. O sistema de terminação a pasto, para animais $1 / 2$ sangue da raça Fla- menga, é viável, porém requer manejo adequado da pastagem para garantir disponibilidade de massa de forragem e proporcionar ganhos de peso elevados aos animais.

\section{Conclusões}

- As características da carcaça e da carne de bovinos $1 / 2$ sangue da raça Flamenga atenderam aos parâmetros de qualidade exigidos pelos frigoríficos no Brasil, independente da suplementação energética com casca de soja;

- A terminação em pastagem de azevém anual cv. Winter Star proporcionou carcaças com pesos elevados, valores adequados de $\mathrm{pH}$, maciez e coloração da carne.

\section{Referências}

AMSA (AMERICAN MEAT SCIENCE ASSOCIATION). Research guidelines for cookery, sensory evaluation and tenderness measurements of fresh meat. 2.ed. Champaing: AMSA, 2015, 105p.

ANDRADE, P.L.; BRESSAN, M.C.; GAMA, L.T; GONÇALVES, T.M.; LADEIRA, M.M; RAMOS, E.M. Qualidade da carne maturada de bovinos Red Norte e Nelore. Revista Brasileira de Zootecnia, v.39, p.1791-1800, 2010. DOI: https://doi.org/10.1590/S151635982010000800023.

Tabela 3. Índices de luminosidade $\left(\mathrm{L}^{*}\right)$, intensidade da cor vermelha ( $\mathrm{a}^{*}$ ) e intensidade da cor amarela $\left(b^{*}\right)$ da carne e da gordura de bovinos mantidos em pastagem de azevém anual, com ou sem suplementação energética ( $0,6 \%$ do peso vivo)

Table 3. Indices of luminosity $\left(L^{*}\right)$, red intensity $\left(a^{*}\right)$ and yellow intensity $\left(b^{*}\right)$ of meat and fat of cattle grazing annual ryegrass pasture, with or without energy supplementation (0.6\% of live weight)

\begin{tabular}{|c|c|c|c|}
\hline Item & Controle & Suplementado & Valor de $p$ \\
\hline \multicolumn{4}{|c|}{ Cor da gordura } \\
\hline$L^{*}$ & $65,2 \pm 1,57$ & $64,3 \pm 1,51$ & 0,678 \\
\hline$a^{*}$ & $4,0 \pm 0,61$ & $5,2 \pm 0,61$ & 0,205 \\
\hline$b^{*}$ & $27,5 \pm 1,85$ & $26,9 \pm 1,77$ & 0,805 \\
\hline \multicolumn{4}{|c|}{ Cor da carne } \\
\hline$L^{*}$ & $36,4 \pm 0,39$ & $36,2 \pm 0,46$ & 0,836 \\
\hline$a^{*}$ & $21,0 \pm 0,68$ & $21,3 \pm 0,66$ & 0,804 \\
\hline$b^{*}$ & $10,1 \pm 0,51$ & $10,2 \pm 0,61$ & 0,850 \\
\hline
\end{tabular}


BATISTA, E.D.; DETMANN, E.; VALADARES FILHO, S.C; TITGEMEYER, E.C; VALADARES, R.F.D. The effect of $C P$ concentration in the diet on urea kinetics and microbial usage of recycled urea in cattle: a meta-analysis. Animal, v.11, p.1303-1311, 2017. DOI: https:// doi.org/10.1017/S1751731116002822.

FERNANDES, A.R.M.; SAMPAIO, A.A.M.; HENRIQUE, W.; OLIVEIRA, E.A.; TULLIO, R.R.; PERECIN, D. Características da carcaça e da carne de bovinos sob diferentes dietas, em confinamento. Arquivo Brasileiro de Medicina Veterinária Zootecnia, v.60, n.1, p.139147, 2008. DOI: https://doi.org/10.1590/ s0102-09352008000100020.

GREGORY, N.G. Animal welfare and meat science. Cambridge: University Press, 1998. $289 p$.

HELLBRUGGE, C. MOREIRA, F.B.; MIZUBUTI, I.Y; PRADO, I.N.; SANTOS, B.P.; PIMENTA, E.P. Desempenho de bovinos de corte em pastagem de azevém (Lolium Multiflorum) com ou sem suplementação energética. Semina: Ciências Agrárias. v.29, p.723-730, 2008.

LAZZAROTTO, E.F.C.O; MENEZES, L.F.G. PARIS, W.; MOLINETE, M.L.; SCHMITZ, G.R.; BARAVIERA, J.H.I.; FARENZENA, R.; PAULA, A.L. Backgrounding steers on temperate grasses mixed with vetch and/or using energy supplementation. Asian-Australasian Journal of Animal Science. v.32, n.6. p.800807, 2019. DOI: https://doi.org/10.5713/ ajas.18.0603 .

MAGGIONI, D. PRADO, I.N.; ZAWADZKI, F.; VALERO, M.V.; MARQUES, J.A.; BRIDI, A.M; MOLETTA, J.L.; ABRAHÃO, J.J.S. Grupos genéticos e graus de acabamento sobre qualidade da carne de bovinos. Semina: Ciências Agrárias, v.33, n.1, p.391 402, 2012. DOI: https://doi.org/10.5433/16790359.2012v33n1p391.

MISSIO, R.L. BRONDANI, I.V.; ALVES FILHO, D.C.; RESTLE, J.; ARBOITTE, M.Z.; SEGABINAZZI, L.R. Características da carcaça e da carne de tourinhos terminados em confinamento, recebendo diferentes níveis de concentrado na dieta. Revista Brasileira de Zootecnia, v.39, p.1610-1617, 2010. DOI: https://doi. org/10.1590/S1516-35982010000700030.

MOLETTA, J.L.; PRADO, I.N.; FUGITA, C.A. EIRAS, C.E.; CARVALHO, C.B.; PEROTTO, D. Características da carcaça e da carne de bovinos não-castrados ou castrados terminados em confinamento e alimentado com três níveis de concentrado. Semina: Ciências Agrárias, v.35, p.1035-1050, 2014. DOI: //doi. org/10.5433/1679-0359.2014v35n2p1035
MOORE, J.E.; BRANT, M.H.; KUNKLE, W.E.; HOPKINS, D.I. Effects of supplementation on voluntary forage intake, diet digestibility, and animal performance. Journal of Animal Science, v.77, p.122-135, 1999. DOI: https:// doi.org/10.2527/1999.77suppl_2122x

MUCHENJE, V.; DZAMA, K.; CHIMONYO, M.; STRYDOM, P.E.; HUGOE, A. RAATS G.J. Some biochemical aspects pertaining to beef eating quality and consumer health: a review. Food Chemistry, v.112, p.279-289, 2009. DOI: https://doi.org/10.1016/j.foodchem.2008.05.103

MULLER, L. Normas para avaliação de carcaças e concurso de carcaça de novilhos. 2.ed. Santa Maria: UFSM. 1987. 31p.

OLIVEIRA, E.A.; SAMPAIO, A.A.M.; HENRIQUE, W.; PIVARO, T.M.; ROSA, B.L.; FERNANDES, A.R.M.; ANDRADE A.T. Quality traits and lipid composition of meat from Nellore young bulls fed with different oils either protected or unprotected from rumen degradation. Meat Science, v.90, p.28-35, 2012. DOI: https://doi.org/10.1016/j.meatsci.2011.05.024

PILAU, A.; ROCHA, M.G.; SANTOS, D.T. Análise econômica de sistemas de produção para recria de bezerras de corte. Revista Brasileira de Zootecnia. v.32, n.4, p.966-976, 2003. DOI: https://doi.org/10.1590/S151635982003000400024.

PITOMBO, R.S.; SOUZA, D.D.N.; RAMALHO, R.O.S.; FIGUEIREDO, A.B.A.; RODRIGUES, V.C.; FREITAS, D.D.G.C.; FERREIRA, J.C.S. Qualidade da carne de bovinos super precoces terminados em confinamento. Arquivo Brasileiro de Medicina Veterinária e Zootecnia, v.65, n.4, p.1203-1207, 2013. DOI: https://doi.org/10.1590/S010209352013000400036

R CORE TEAM (2018). R: A language and environment for statistical computing. R Foundation for Statistical Computing, Vienna, Austria. URL https://www.R-project.org/.

REALINI, C. E.; DUCKETT, S.K.; BRITO, G.W.; DALLA RIZZA, M.; MATTOS, D. Effect of pasture vs. concentrate feeding with or without antioxidants on carcass characteristics, fatty acid composition, and quality of Uruguayan beef. Meat Science, v.66, n.3, p.567-577, 2004. DOI: https://doi.org/10.1016/S03091740(03)00160-8.

RESENDE, F.D; GESUALDI JÚNIOR, A; QUEIROZ, A.C.; FARIA, M.H; VIANA, A.P. Carcass characteristics of feedlot-finished Zebu and Caracu cattle. Revista Brasileira de Zootecnia, v. 43, p. 67-72, 2014. DOI: https://doi.
org/10.1590/S1516-35982014000200003.

RESTLE, J. VAZ, F.N.; ALVES FILHO, D.C.; PASCOAL, L.L.; OLIVEIRA, A.N.; FATURI, C.; ARBOITTE, M.Z. Efeito da suplementação energética sobre a carcaça de vacas de diferentes idades, terminadas em pastagem cultivada de estação fria sob pastejo horário. Revista Brasileira de Zootecnia, v.30, n.3, p.10761083, 2001. DOI: https://doi.org/10.1590/ S1516-35982001000400023.

RIBEIRO, F.G.; LEME, P.R.; BULLE, M.L.M.; LIMA, C.G.; SILVA, S.L.; PEREIRA, A.S.C.; LANNA, D.P.D. Características da carcaça e qualidade da carne de tourinhos alimentados com dietas de alta energia. Revista Brasileira de Zootecnia, v.31, p.749-756, 2002. DOI: https://doi.org/10.1590/S151635982002000300026.

ROCHA, C.E. Fatores que influenciam características e valor da carcaça em um rebanho de bovinos da raça Nelore. 1999. 95p. Dissertação (Mestrado em Zootecnia) - Curso de Pós-graduação em Zootecnia, Universidade Estadual Paulista.

RODRIGUES, D.A.; AVANZA, M.F.B.; DIAS L.G.G. Sobressemeadura de Aveia e Azevém em Pastagens Tropicais no Inverno - Revisão de Literatura. Revista Científica Eletrônica de Medicina Veterinária. v.9, n.16, 2011.

SANTOS, D.T.; ROCHA, M.G; QUADROS, F.L.F.; GENRO, T.C.M.; MONTAGNER, D.B; GONÇALVES, E.N.; ROMAN, J. Suplementos Energéticos para Recria de Novilhas de Corte em Pastagens Anuais. Desempenho Animal. Revista Brasileira de Zootecnia, v.34, n.1, p.209-219, 2005. DOI: ?https://doi.org/10.1590/S151635982005000100025.

SILVEIRA, M.F.; KOZLOSKI, G.V.; BRONDANI, I.L.; ALVES FILHO, D.C; RESTLE, J.; LEITE, D.T.; METZ, P.A.M.; SILVEIRA, S.R.L. Ganho de peso vivo e fermentação ruminal em novilhos mantidos em pastagem cultivada de clima temperado e recebendo diferentes suplementos. Ciência Rural, v.36, n.3, p.898903, 2006. DOI: https://doi.org/10.1590/ S0103-84782006000300026.

TAMBARA, A.A.C. OLIVO, C.J; PIRES, M.B.G.; SANCHEZ, L.M.B. Avaliação in vivo da digestibilidade da casca do grão de soja moída com ovinos. Revista Ciência Rural, v.25, n.2, p.283-287, 1995. DOI: https://doi. org/10.1590/S0103-84781995000200019

TAPP III, W.N.; YANCEY; J.W.S.; APPLE, J.K. How is the instrumental color of meat measured? Meat Science, v.89, p.1-5, 2011. DOI: $\quad$ https://doi.org/10.1016/j.meatsci.2010.11.021 